\section{Extent of silent cerebral infarcts in adult sickle-cell disease patients on magnetic resonance imaging: is there a correlation with the clinical severity of disease?}

\author{
Ekaterini Solomou, ${ }^{1}$ Pantelis Kraniotis, ${ }^{1}$ \\ Alexandra Kourakli, ${ }^{2}$ Theodore Petsas ${ }^{1}$ \\ Departments of ${ }^{1}$ Radiology and \\ ${ }^{2}$ Hematology, University Hospital \\ of Patras, Greece
}

\begin{abstract}
The aim of this paper is to correlate the extent of silent cerebral infarcts (SCIs) on magnetic resonance imaging (MRI) with the clinical severity of sickle cell disease (SCD) in adult patients. Twenty-four consecutive adult asymptomatic SCD patients (11 male and 13 female) with a mean age of 38.4 years (range 20-59) were submitted to brain MRI on a 1 Tesla Gyroscan Intera, Philips MR scanner with a dedicated head coil. The protocol consisted of TSE T2-weighted and FLAIR images on the axial and coronal planes. MRI readings were undertaken by two radiologists and consensus readings. Patients were compound heterozygotes $(\mathrm{HbS} / \beta$-thal). The extent of SCIs was classified from $0-2$ with 0 designating no lesions. Clinical severity was graded as $0-2$ by the hematologist, according to the frequency and severity of vaso-occlusive crises. There was no statistically significant correlation between the severity of clinical disease and the extent of SCIs on MR imaging. The extent of SCI lesions did not differ statistically between younger and older patients. Patients receiving hydroxyurea had no statistically significant difference in the extent of SCI lesions. The extent of SCIs in heterozygous $(\mathrm{HbS} / \beta$-thal) SCD patients is not age related and may be quite severe even in younger $(<38.4$ years) patients. However the extent of SCIs is not correlated with the severity of clinical disease.
\end{abstract}

\section{Introduction}

Sickle cell disease (SCD) is a genetic hemoglobinopathy, in which hemoglobin, is slightly abnormal. ${ }^{1}$ Cerebral damage is a serious complication in SCD patients, ${ }^{2}$ with cerebral infarction (overt stroke) secondary to occlusive vasculopathy developing in $5.5-17 \%$ of patients with SCD. ${ }^{3,4}$ Conversely the inci- dence of silent cerebral infarcts (SCIs) lies between $3-38 \%$ in patients with beta-thalassemia and between 5-31\% in patients with $\mathrm{HbSC}^{5-10}$ being far more common in this patient population.

The pathophysiology of cerebrovascular disease in sickle cell anemia is probably related to two processes: i) intimal hyperplasia and ii) thrombosis.,11,12 Stroke is attributed to both microvascular and macrovascular lesions.

According to the microvascular scenario the small vessels are more likely to be involved in cases of peripheral vaso-occlusive events. In such cases the arteriole and postcapillary venule are the major sites of sicklecell adhesion and subsequent occlusion. The deep white matter, which is perfused by arterioles with sparse anastomoses, is more susceptible to inadequate perfusion and infarction. The deoxygenated, less flexible sickled red blood cells, adhere to the endothelium of capillaries and venules, leading to intravascular stasis and microvascular occlusion. ${ }^{13}$

In the macrovascular scenario, SCD patients develop chronic anemia resulting in hyperdynamic blood circulation and increased velocity of the blood flow, which has severe consequences on the vessels. ${ }^{14}$ Repeated red blood cell (RBC) adhesion, followed by forcible removal under high-shear forces causes endothelial injury, leading to intimal hyperplasia and endoluminal narrowing. Subsequently RBC adherence to damaged vessel walls results in secondary thrombosis, occlusion of the vessel and distal branch emboli. ${ }^{13}$ The effects of these lesions depend on the vessel involved, as well as the rapidity of the process. Approximately $75 \%$ of strokes in SCD patients are the result of occlusion of large arteries. ${ }^{15}$

However, as stated earlier, the most common form of CNS damage in SCD patients is silent cerebral infarct (SCI). The definition of SCI comprises both an abnormal MRI with absence of physical findings of overt stroke. ${ }^{2}$ Surprisingly SCD patients will have an incidence of SCI up to $27 \%$ by age 6 and $37 \%$ by age 14. ${ }^{16,17}$ Recent evidence suggests that adult SCD patients are prone to develop new and ongoing SCIs. ${ }^{2}$ The latter may on the one hand be the cause for poor intellectual performance in these patients and on the other hand either become progressive SCI or overt stroke disease. The first study addressing the issue of SCI was undertaken with CT and patients were referred to be suffering a covert stroke. ${ }^{18}$ Subsequently and after the Cooperative Study of Sickle Cell Disease (CSSCD) the term SCI or silent stroke is preferred. ${ }^{19}$

The aim of the study is to find out if there is any correlation between the extent of silent cerebral infartcs (SCIs) depicted on MRI with the disease severity in SCD adult patients.
Correspondence: Pantelis Kraniotis, University Hospital of Patras, Department of Radiology, Rion 26500, Patras, Greece.

Tel/Fax: +30.261.3603215.

E-mail: pantelis.kraniotis@gmail.com

Key words: sickle cell disease, heterozygous state, magnetic resonance imaging, silent cerebral infarcts.

Contributions: the authors contributed equally.

Conflict of interests: the authors declare no conflict of interests.

Received for publication: 12 September 2012.

Revision received: 11 December 2012.

Accepted for publication: 7 January 2013.

This work is licensed under a Creative Commons Attribution NonCommercial 3.0 License (CC BYNC 3.0).

(C) Copyright E. Solomou et al., 2013

Licensee PAGEPress, Italy

Hematology Reports 2013; 5:e3

doi:10.4081/hr.2013.e3

\section{Materials and Methods}

\section{Study group and clinical evaluation}

The study group consisted of 24 consecutive patients (11 men, 13 women) with known SCD and a mean age of 38.4 (range 20-59) years. The study was approved by the local ethical committee and informed consent was obtained prior to each examination from all patients. Patients were compound heterozygotes $(\mathrm{HbS} / \beta$-thal). All patients included in the study had no evidence of focal neurologic deficit from physical examination. Patients with any clinical evidence of neurologic deficit were excluded from the study, as well as patients with contraindications to MR imaging, such as the presence of cardiac pacemakers or metallic implants.

The disease was classified according to the number of vaso-occlusive episodes which needed hospitalization per year, the number of transfused RBC units per year, the degree of hemolysis, and the incidence of acute chest syndrome (ACS), pulmonary hypertension (PH) and acute splenic sequestration (ASS). A patient with more than 6 hospitalizations per year and/or for more than 30 days in total, or transfusion-dependency with more than 2 units RBC per month, or with a continuously high degree of intravascular hemolysis, or finally with a history of ACS, PH, ASS, was considered as severely ill (grade 2). The disease of a patient with none of the above criteria was characterized as mild (grade 0 ), whereas the presence of 3-5 hospitalizations per year for 
less than 30 days or sporadic transfusions as moderately severe (grade 1).

Treatment with Hydroxyurea was applied to patients with severe and moderately severe form of the disease, and it clearly changed disease severity, dramatically. However, for the needs of this study, the definition of disease severity was based on the patient's history since birth, and it did not take into consideration the last period on Hydroxyurea therapy.

From these patients (50\%) 12/24 had a history of splenectomy. $66.67 \%(16 / 24)$ of the patients were on treatment with hydroxyurea, due to history of more frequent or pronounced vaso-occlusive episodes.

\section{Magnetic resonance imaging protocol and imaging evaluation}

All patients were submitted to brain MRI on a 1 Tesla, Gyroscan Intera, Philips MR scanner with a dedicated head coil. MRI protocol consisted of TSE T2-weighted (TR $5300 \mathrm{~ms}$, TE 120 $\mathrm{ms}$ ) and FLAIR (TR $6000 \mathrm{~ms}$, TE $110 \mathrm{~ms}$, TI $2000 \mathrm{~ms}$ ) images in the axial and coronal planes. Imaging parameters included slice thickness of $5 \mathrm{~mm}$ and matrix size of 189x256.

The examinations were reviewed by two radiologists, which were blinded to the clinical results, for evidence of SCIs (i.e. lacunar infarcts and leukoencephalopathy).

Lacunar infarcts were defined as focal (less than $1 \mathrm{~cm}$ ) high intensity lesions on the T2weighted or FLAIR images.

Leukoencephalopathy was defined as multiple high intensity lesions over $1 \mathrm{~cm}$ on the T2weighted or FLAIR images.

Score 0 was attributed to patients with normal findings.

Score 1 was attributed to small unifocal lesions $<1 \mathrm{~cm}$ in diameter or mild extent leukoencephalopathy with lesions measuring $<3 \mathrm{~cm}$.

Score 2 was attributed to more severe/diffuse leukoencephalopathy with lesions $>3 \mathrm{~cm}$.

The final score, according to the previous definitions was reached by consensus readings.

Irrespective of their SCI score, patients were also evaluated for evidence of brain atrophy, with binary criteria either existing or nonexisting. Atrophy was defined as lesser volume of brain tissue than the one expected in a healthy person of similar age.

\section{Results}

Based on clinical criteria 33.33\% (8/24) of the patients had mild, $37.50 \%$ (9/24) moderate and $29.17 \%$ (7/24) severe clinical disease.

Abnormalities were evident on MRI in $66.67 \%(16 / 24)$ of the patients. More specifically $8.33 \%(2 / 24)$ of them were classified as score 1 and $58.33 \%(14 / 24)$ were classified as score 2 (Figure 1A-C).

The rest $33.3 \%$ (8/24) of the patients had no evidence of cerebral lesions on MRI and were classified as score 0 . From these 8 patients with no abnormalities on the MRI, $25 \%$ (2/8) had mild, $37.50 \%(3 / 8)$ had moderate and the remaining $37.50 \%(3 / 8)$ had severe clinical disease.

From the 2 patients with score 1 on MRI, $50 \%(1 / 2)$ had moderate clinical disease and $50 \%(1 / 2)$ had severe clinical disease. From the 14 patients with score 2 on the MRI, $42.86 \%$ (6/14) of them had mild, $35.71 \%$ (5/14) moderate and $21.43 \%(3 / 14)$ had severe clinical disease. The results are summarized in Table 1.

Atrophy was found in $54.16 \%$ (13/24) of the patients (Figure 2A, B).

Clinical severity and MRI score were investigated both through the Spearman's correlation coefficient (rho=-0.227, $\mathrm{P}=0.286$ ) and with tests for independence $(\mathrm{P}=0.72$, chisquare test). No statistically significant corre- lation between them was observed.

Considering the 38.4 years as the mean age, the patients were subcategorized in two groups: the younger group whose age was under 38.4 years and the older group aged over 38.4. In the younger age group $35.71 \%$ (5/14) did not show any abnormalities on the MRI and were classified as score 0, no patients were classified as score 1 and $64.29 \%$ (9/14) of them were score 2. Alternatively from the older age group 30\% (3/10) did not exhibit any abnormalities and were classified as score $0,20.00 \%(2 / 10)$ patients were classified as score 1 , whereas $50 \%$ (5/10) were classified as score 2, on MRI. The observed difference in MRI scores between the two age groups was not statistically significant ( $\mathrm{P}=0.216$, chi-square test). The results are summarized in Table 2. Moreover the MRI score was not correlated either with patient gender $(\mathrm{P}=0.950)$ or with hydroxyurea treatment $(\mathrm{P}=0.230)$.
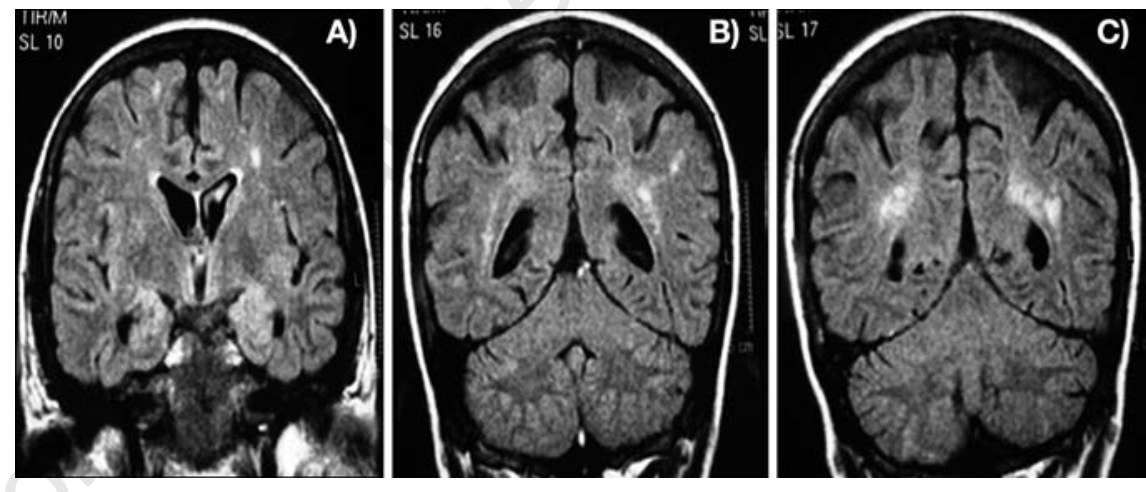

Figure 1A-C. Coronal fluid-attenuated inversion recovery images from anterior to posterior. A 44-year-old patient with multifocal lesions of the white matter scored as grade 2 .
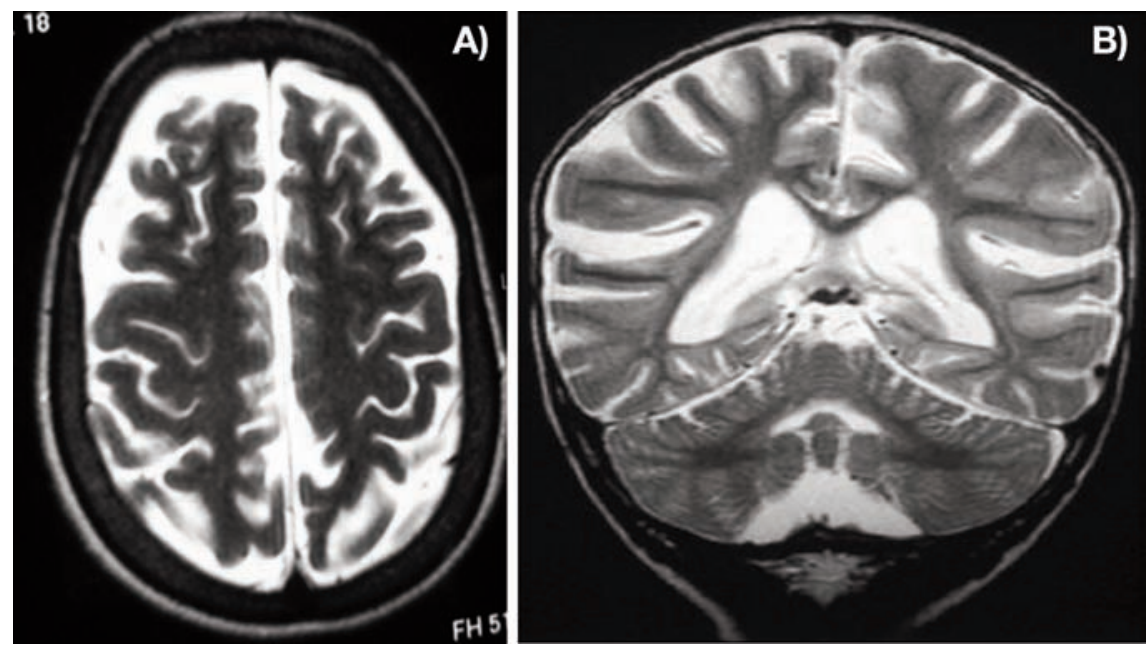

Figure 2. Axial (A) and coronal (B) T2-WI. Cerebral atrophy in a 20-year-old sickle cell disease patient (A) and in a 22-year-old sickle cell disease patient (B). 


\section{Discussion}

Sickle cell disease (SCD) is characterized by hemolytic anemia, an increased susceptibility to infections, growth retardation, painful crises, acute chest syndrome and vaso-occlusion. The latter occurs in almost all vascular beds, resulting in ischemia, including the central nervous system, with high morbidity and mortality. Outcome is difficult to predict and few effective treatments are available. ${ }^{1}$

Stroke is a serious complication in patients with sickle cell disease occurring in 5-17\%, most often between 9 to 15 years of age. ${ }^{14}$ There are two main theories for brain infarcts in these patients including both a microvascular scenario and a macrovascular scenario.

Recent research indicates that SCD is also associated with silent cerebral infarcts (SCIs). The latter is defined as the presence of abnormalities on neuroimaging studies, in the absence of overt clinical symptoms. ${ }^{20} \mathrm{SCIs}$ are thought to rather represent small vessel disease but direct evidence is still lacking. ${ }^{2}$ Furthermore SCIs seem to affect more the borderline zones and this may suggest hemodynamic reasons implicated in its pathogenesis. ${ }^{2}$ MRI has proved to be highly sensitive in the identification of SCIs. Due to its sensitivity to detect increased amounts of water within brain tissue at sites of acute and chronic infarctions, especially with fluid-attenuated inversion recovery (FLAIR) images, MRI has revolutionized the ability to see not only acute symptomatic infarctions, but also SCIs, especially those within the deep white matter of the centrum semiovale. ${ }^{1}$

In our study a total $66.67 \%(16 / 24)$ of the patients had evidence of SCIs. In contrast in the CSSCD cohort the prevalence of SCIs at baseline was $21.2 \%,{ }^{10}$ while in the SIT trial the presence of SCIs reached a total of $30.8 \% .^{2}$ Both of these studies however have included children and teenagers. The overall percentage of reported SCI incidence in the literature is between 3$38 \%$ in patients with beta-thalassemia and between $5-31 \%$ in patients with $\mathrm{HbSC}$. ${ }^{-10}$

SCIs are known to be more common in homozygous SS patients but they are also known to happen to heterozygous patients like sickle beta-thalassemia and $\mathrm{HbSC}$ disease. While one would probably expect a lower overall incidence of SCIs in our heterozygous SCD patients this was surprisingly not the case as the incidence was far greater than that reported in the literature for heterozygous SCD adult patients. More specifically a study by Kugler $e t$ $a .^{21}$ found that $50 \%$ of the homozygous SDC adult patients (mean age 20 years) had SCIs and of these lesions $16 \%$ were at least $1.5 \mathrm{~cm}$. Vichinsky et al. ${ }^{22}$ reported an SCI incidence of $13 \%$ in his study with adult (19-55 years) homozygous SCD patients. The overall percentage of SCIs found by Marouf et al. ${ }^{19}$ comprising adult patients with a mean age of $26.9 \pm 9.3$ years with both homozygous SS and heterozygous $\mathrm{S} \beta^{0}$ Thal was $20 \%$. Manfre et al. ${ }^{6}$ studied adult patients younger than 50 years with thalassemia intermedia and homozygous sickle-cell thalassemia. They found SCIs in $37.5 \%$ in patients with thalassemia intermedia and $52 \%$ in patients with homozygous sickle cell-thalassemia disease.

Differences in results between studies may reflect study population differences and differences concerning the study protocol and definitions. There seems to be different ways to define SCIs and there is no wider consensus. ${ }^{23}$ For example in the Silent Cerebral Infarct Trial (SIT trial) $^{24}$ it included lesions that were at least $3 \mathrm{~mm}$ in their greatest dimension and visible in at least two planes on the T2-weighted images. In the study by Vichinsky et $a .^{22}$ evaluating adult patients the definition of SCI included lesions of at least $5 \mathrm{~mm}$, hyperintense on the T2-weighted image but had to be hypointense on the T1-weighted image. According to Zhu et al. ${ }^{23}$ discrepancies in the literature are mainly due to: ${ }^{23}$ i) differences in MR parameters, like slice thickness, ii) different criteria for SCI definition and iii) Different criteria for defining dilated Virchow-Robin spaces (dVRS).

While a high percentage $58.33 \%$ (14/24) of our patients had a high MRI score of 2 , only a small percentage $29.17 \%$ (7/24) of these patients had severe disease according to clinical criteria. Manrfe et al. ${ }^{6}$ found that brain damage was more severe in patients with sickle cell-thalassemia disease who had more vaso-occlusive episodes per year. This was not

the case in our study, where the extent of SCIs was not well-correlated with clinical severity. However according to other previous studies, ${ }^{25}$ various imaging techniques reveal a more extensive and diffuse pattern of vascular involvement than the one expected from the patient's clinical presentation. This may imply the silent, insidious nature and complex pathophysiology of microvascular disease in SCD patients. In the study of Vichinski et al. ${ }^{22}$ lacunae (lesions $\leq 5 \mathrm{~mm}$ ) were seen in $13 \%$ and white matter lesions in $15 \%$ and in our study lacunar lesions (5-9 mm) were seen in $8.33 \%$ and larger lesions ( $>10 \mathrm{~mm}$ ) in $58.33 \%$. Although the study design is different it seems that while moderate MRI disease scores are rather similar in both studies, severe MRI disease scores are much higher in our study.

In our study younger and older patient groups did not show statistically significant differences in the extent of SCIs. More specifically a high percentage $64.26 \%(9 / 14)$ from the patients of the younger age group $(<38.4$ years) showed SCIs and interestingly all of them were graded score 2 , which would be unusual for the general population of similar age. In the older ( $>38.4$ years) age group patients exhibited SCIs in a total percentage of $70 \%(7 / 10)$, whereas 20\% (2/10) were score 1 and $50 \%(5 / 10)$ were scored 2 on MRI. In contrast, Manrfe et $a l .{ }^{6}$ reported that in thalassemia intermedia patients, the frequency of brain damage increased with age.

It is known that normal adults accumulate hyperintensities while ageing. Previous estimates have shown that the incidence of SCI with MRI in the general population is between $5.8 \%$ and $17.7 \%{ }^{26,27}$ More specifically the incidence of these hyperintensities has been

Table 1. Distribution of silent cerebral infarcts in patients with sickle cell disease.

\begin{tabular}{lcccc} 
MIRI score & \multicolumn{4}{c}{ Clinical severity of disease } \\
& Mild disease & Moderate disease & Severe disease & Total \\
Score 0 & $2 / 24$ & $3 / 24$ & $3 / 24$ & $8 / 24(33.33 \%)$ \\
Score 1 & $0 / 24$ & $1 / 24$ & $1 / 24$ & $2 / 24(8.33 \%)$ \\
\hline Score 2 & $6 / 24$ & $5 / 24$ & $3 / 24$ & $14 / 24(58.33 \%)$ \\
Total & $8 / 24(33.33 \%)$ & $9 / 24(37.50 \%)$ & $7 / 24(29.17 \%)$ & \\
\hline
\end{tabular}

MRI, magnetic resonance imaging.

Table 2. Distribution of silent cerebral infarcts in the two age groups.

\begin{tabular}{lcc} 
MIRI score & \multicolumn{3}{c}{$\begin{array}{c}\text { Age groups } \\
\text { Younger age group }<38.4\end{array}$} \\
Score 0 & $5 / 14(35.71 \%)$ & $3 / 14(30.00 \%)$ \\
Score 1 & $0 / 24$ & $2 / 14(20.00 \%)$ \\
\hline Score 2 & $9 / 14(64.29 \%)$ & $5 / 14(50.00 \%)$ \\
Total & $14 / 24$ & $10 / 24$ \\
\hline
\end{tabular}

MRI, magnetic resonance imaging. 
$15.6 \%$ and $10.7 \%$ in two large studies. ${ }^{28,29}$ The Framingham offspring study found an incidence or $10.7 \%,{ }^{28}$ using 1 Tesla MRI and $4 \mathrm{~mm}$ section thickness in a patient population with a mean age of $62 \pm 9$ years. ${ }^{28}$ On the other hand the incidence was $15.6 \%$ in the Helsinki Aging Brain Study, ${ }^{29}$ using patients between 55 and 85 years and a $0.2-\mathrm{T}$ MRI and $10 \mathrm{~mm}$ section thickness. In our case a study limitation factor may be the fact that there were no agematched controls. This may, however be counteracted by the fact that the younger patient's group $<38.4$ years still showed a significant extent of SCIs. The fact that there was no statistically significant difference between the two age groups, in our patients, implies that SCIs in adult heterozygous SCD patients may not be age-related and may start and accumulate from younger ages. This may be of clinical significance, as presence of SCIs may result in further brain damage as they are known to progress to symptomatic disease. In fact results from the CSSCD study indicate that SCI poses further risk for extra neurological damage and a higher risk for both progressive SCI or overt clinical stroke. ${ }^{2}$

Furthermore, brain atrophy was commonly observed in our patients, as it occurred in $54.16 \%(13 / 24)$ of the whole population studied. Our results show a higher rate of brain atrophy than those reported in the literature. Silva et al reported a lower incidence of brain atrophy (28\%) in SDC patients, aged 16 years and above.$^{30}$ In the Manfre et al. ${ }^{6}$ study atrophy was detected in $31 \%$ in the thalassemia intermedia group, and reached $51 \%$ in the homozygous SCD group. In the study by Vichinski $e t$ $a l^{22}$ atrophy was seen in 23\%. Atrophy in SCD patients is considered to suggest a chronic underlying process. ${ }^{2}$

In the literature, the risk factors for SCI are reported in the CSSCD study as being history of seizures, pain event rate, elevated WBC and the SEN betas globin gene haplotype. ${ }^{31}$ In the SIT trial risk factors for SCI included: ${ }^{24}$ hemoglobin $<7.6 \mathrm{~g} / \mathrm{dL}$, systolic blood pressure $>113$ $\mathrm{mmHg}$ and male gender. In our study though, male gender was not a factor for higher SCI prevalence.

Hydroxyurea and hematopoietic stem cell transplantation are considered for primary and secondary prevention of SCI but there is still no high level evidence to support this. ${ }^{2}$ In our study there were no statistically significant differences in the extent of SCIs between patients receiving or not receiving hydroxyurea. Some other study limitations in comparison to other studies are: the rather small number of patients studied and the use of 1T MRI scanner instead of the 1.5T used in other studies. According to the field strength and study parameters (slice thickness of $5 \mathrm{~mm}$ ) the smallest lesions that could be picked-up, would be around $5 \mathrm{~mm}$. We did not use lesion subcategorization for lesions $<10 \mathrm{~mm}$ but instead of it all lesions $>5 \mathrm{~mm}$ and $<10 \mathrm{~mm}$ were given score 1 . This may have made it more difficult to compare our results with those of larger studies.

The clinical criteria for disease severity were rather qualitative, but all patients were on follow-up for many years, as the Thalassemia and Hemoglobinopathies Unit of our hospital is a reference center for SCD patients.

Concerning the incidence of brain atrophy it should be noted that the definition is rather subjective and this may also create bias when comparing with previous studies. This is reflected in previous studies with the reported incidence being between $23-51 \%$

Finally it should be noted that although it is known that SCI may occur in infants just 1 year of age and continue during childhood, reaching maximum incidence rate in children around 6 years of age, little is known about the natural history of SCIs in the adult, which is not thoroughly understood. ${ }^{2}$ Our study may further contribute to the understanding of the disease in the adult as it is one of a few focusing on heterozygous sickle-cell/ $\beta$-thal adult patients. ${ }^{6,7,21,22}$ in contrast to larger cohorts that focus on homozygous SCD patients of school-age or teenager patients.

\section{Conclusions}

In conclusion, the extent of silent cerebral infarcts on MR imaging in compound heterozygous (HBS/ $\beta$-thal) adult patients is not correlated with the severity of clinical disease. Moreover the extent of SCIs on MR imaging in these patients is not age-related and may be quite severe even in the younger ones.

\section{References}

1. Schnog JB, Duits AJ, Muskiet FA, et al. Sickle cell disease; a general overview. Neth J Med 2004;62:364-74.

2. Debaun MR, Armstrong FD, McKinstry RC, et al. Silent cerebral infarcts: a review on a prevalent and progressive cause of neurologic injury in sickle cell anemia. Blood 2012;119:4587-96.

3. Ohene-Frempong K, Weiner SJ, Sleeper LA, et al. Cerebrovascular accidents in sickle cell disease: rates and risk factors. Blood 1998;91:288-94.

4. Seibert JJ, Glasier CM, Kirby RS, et al. Transcranial doppler, MRA, and MRI as a screening examination for cerebrovascular disease in patients with sickle cell ane- mia: an 8-year study. Pediatr Radiol 1998;28:138-42.

5. Bernaudin F, Verlhac S, Freard F, et al. Multicenter prospective study of children with sickle cell disease: radiographic and psychometric correlation. J Child Neurol 2000;15:333-43.

6. Manfre L, Giarratano E, Maggio A, et al. MR imaging of the brain: findings in asymptomatic patients with thalassemia intermedia and sickle cell-thalassemia disease. AJR Am J Roentgenol 1999;173: 1477-80.

7. Marouf R, Gupta R, Haider MZ, Adekile AD. Silent brain infarcts in adult Kuwaiti sickle cell disease patients. Am J Hematol 2003;73:240-3.

8. Zafeiriou DI, Prengler M, Gombakis N, et al. Central nervous system abnormalities in asymptomatic young patients with Sbetathalassemia. Ann Neurol 2004;55: 835-9.

9. Armstrong FD, Thompson RJ Jr., Wang W, et al. Cognitive functioning and brain magnetic resonance imaging in children with sickle Cell disease. Neuropsychology Committee of the Cooperative Study of Sickle Cell Disease. Pediatrics 1996;97: 864-70.

10. Pegelow CH, Macklin EA, Moser FG, et al. Longitudinal changes in brain magnetic resonance imaging findings in children with sickle cell disease. Blood 2002;99: 3014-8.

11. Seibert JJ, Miller SF, Kirby RS, et al. Cerebrovascular disease in symptomatic and asymptomatic patients with sickle cell anemia: screening with duplex transcranial Doppler US-correlation with MR imaging and MR angiography. Radiology 1993;1892:457-66.

12. Steen RG, Emudianughe T, Hankins GM, et al. Brain imaging findings in pediatric patients with sickle cell disease. Radiology 2003;228:216-25.

13. Kandeel AY, Zimmerman RA, OheneFrempong K. Comparison of magnetic resonance angiography and conventional angiography in sickle cell disease: clinical significance and reliability. Neuroradiology 1996;38:409-16.

14. Adams RJ, Ohene-Frempong K, Wang W. Sickle cell and the brain. Hematology Am Soc Hematol Educ Program 2001:31-46.

15. Moran CJ, Siegel MJ, DeBaun MR. Sickle cell disease: imaging of cerebrovascular complications. Radiology 1998;206:311-21.

16. Kwiatkowski JL, Zimmerman RA, Pollock AN, et al. Silent infarcts in young children with sickle cell disease. Br J Haematol 2009;146:300-5.

17. Bernaudin F, Verlhac S, Arnaud C, et al. Impact of early transcranial Doppler screening and intensive therapy on cerebral vasculopathy outcome in a newborn 
sickle cell anemia cohort. Blood 2011;117: 1130-40.

18. Hindmarsh PC, Brozovic M, Brook CG, Davies SC. Incidence of overt and covert neurological damage in children with sickle cell disease. Postgrad Med J 1987;63: 751-3.

19. Moser FG, Miller ST, Bello JA, et al. The spectrum of brain MR abnormalities in sickle-cell disease: a report from the Cooperative Study of Sickle Cell Disease. AJNR Am J Neuroradiol 1996;17:965-72.

20. Steen RG, Hankins GM, Xiong X, et al. Prospective brain imaging evaluation of children with sickle cell trait: initial observations. Radiology 2003;228:208-15.

21. Kugler S, Anderson B, Cross D, et al. Abnormal cranial magnetic resonance imaging scans in sickle-cell disease. Neurological correlates and clinical implications. Arch Neurol 1993;50:629-35.

22. Vichinsky EP, Neumayr LD, Gold JI, et al. Neuropsychological dysfunction and neu- roimaging abnormalities in neurologically intact adults with sickle cell anemia. JAMA 2010;303:1823-31.

23. Zhu YC, Dufouil C, Tzourio C, Chabriat H. Silent brain infarcts: a review of MRI diagnostic criteria. Stroke 2011;42:1140-5.

24. Casella JF, King AA, Barton B, et al. Design of the silent cerebral infarct transfusion (SIT) trial. Pediatr Hematol Oncol 2010;27:69-89.

25. Prengler M, Pavlakis SG, Boyd S, et al. Sickle cell disease: ischemia and seizures. Ann Neurol 2005;58:290-302.

26. Lee SC, Park SJ, Ki HK, et al. Prevalence and risk factors of silent cerebral infarction in apparently normal adults. Hypertension 2000;36:73-7.

27. Price TR, Manolio TA, Kronmal RA, et al. Silent brain infarction on magnetic resonance imaging and neurological abnormalities in community-dwelling older adults. The Cardiovascular Health Study. CHS Collaborative Research Group. Stroke
1997;28:1158-64.

28. Das RR, Seshadri S, Beiser AS, et al. Prevalence and correlates of silent cerebral infarcts in the Framingham offspring study. Stroke 2008;39:2929-35.

29. Ylikoski A, Erkinjuntti T, Raininko R, et al. White matter hyperintensities on MRI in the neurologically nondiseased elderly. Analysis of cohorts of consecutive subjects aged 55 to 85 years living at home. Stroke 1995;26:1171-7.

30. Silva GS, Vicari P, Figueiredo MS, et al. Brain magnetic resonance imaging abnormalities in adult patients with sickle cell disease: correlation with transcranial Doppler findings. Stroke 2009;40:2408-12.

31. Kinney TR, Helms RW, O'Branski EE, et al. Safety of hydroxyurea in children with sickle cell anemia: results of the HUGKIDS study, a phase I/II trial. Pediatric Hydroxyurea Group. Blood 1999;94:1550-4. 